\title{
The Photodetector Array Camera and Spectrometer (PACS) for the Herschel Space Observatory
}

\author{
Albrecht Poglitsch ${ }^{a}$, Christoffel Waelkens ${ }^{b}$, Otto H. Bauer ${ }^{a}$, Jordi Cepa ${ }^{c}$, \\ Helmut Feuchtgruber ${ }^{a}$, Thomas Henning ${ }^{d}$, Chris van Hoof ${ }^{e}$, Franz Kerschbaum ${ }^{f}$, \\ Oliver Krause $^{d}$, Etienne Renotte ${ }^{g}$, Louis Rodriguez ${ }^{h}$, Paolo Saraceno ${ }^{i}$, and \\ Bart Vandenbussche ${ }^{b}$ \\ ${ }^{a}$ Max-Planck-Institut für extraterrestrische Physik, Postfach 1312, 85741 Garching, Germany \\ ${ }^{b}$ Katholieke Universiteit Leuven, Celestijnenlaan 200B, 3001 Leuven, Belgium \\ ${ }^{c}$ Instituto de Astrofisica de Canarias, C/Via Lactea s/n, La Laguna, \\ 38200 Santa Cruz de Tenerife, Spain \\ ${ }^{d}$ Max-Planck-Institut für Astronomie, Königstuhl 17, 69117 Heidelberg, Germany \\ ${ }^{e}$ Interuniversity Microelectronics Center, Kapeldreef 75, 3001 Leuven, Belgium

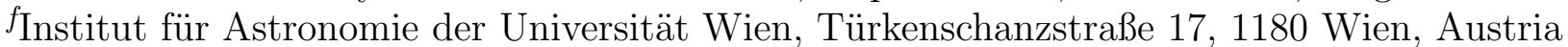 \\ ${ }^{g}$ Centre Spatial de Liège, Parc Scientifique du Sart Tilman, Avenue du Pré-Aily, \\ 4031 Angleur-Liège, Belgium \\ ${ }^{h}$ Commissariat à l'Energie Atomique, Service d'Astrophyisque, Orme des Merisiers, Bât. 709, \\ 91191 Gif/Yvette, France \\ ${ }^{i}$ Istituto di Fisica dello Spazio Interplanetario, Via del Fosso del Cavaliere, 00133 Roma, Italy
}

\begin{abstract}
The Photodetector Array Camera and Spectrometer (PACS) is one of the three science instruments for ESA's far infrared and submillimeter observatory Herschel. It employs two Ge:Ga photoconductor arrays (stressed and unstressed) with $16 \times 25$ pixels, each, and two filled silicon bolometer arrays with $16 \times 32$ and $32 \times 64$ pixels, respectively, to perform imaging line spectroscopy and imaging photometry in the $60-210 \mu \mathrm{m}$ wavelength band. In photometry mode, it will simultaneously image two bands, $60-85 \mu \mathrm{m}$ or $85-125 \mu \mathrm{m}$ and $125-210 \mu \mathrm{m}$, over a field of view of $\sim 1.75^{\prime} \times 3.5^{\prime}$, with close to Nyquist beam sampling in each band. In spectroscopy mode, it will image a field of $\sim 50 " \times 50$ ", resolved into $5 \times 5$ pixels, with an instantaneous spectral coverage of $\sim 1500 \mathrm{~km} / \mathrm{s}$ and a spectral resolution of $\sim 175 \mathrm{~km} / \mathrm{s}$. In both modes the performance is expected to be not far from background-noise limited, with sensitivities ( $5 \sigma$ in $1 \mathrm{~h}$ ) of $\sim 4 \mathrm{mJy}$ or $3-20 \times 10^{-18} \mathrm{~W} / \mathrm{m}^{2}$, respectively.

We summarize the design of the instrument, describe the observing modes in combination with the telescope pointing modes, report results from instrument level performance tests and calibration of the Flight Model, and present our current prediction of the in-orbit performance of the instrument based on the ground tests.
\end{abstract}

Keywords: Instruments: far infrared; instruments: space; instruments: photometer, integral field spectrometer; missions: Herschel

\section{INTRODUCTION}

The far infrared and submillimetre satellite Herschel will open up the wavelength range $60-600 \mu \mathrm{m}$ to photometry and spectroscopy with unprecedented sensitivity and spatial resolution, unobscured by the Earth's atmosphere.

Within the complement of three instruments selected to form the science payload, the shortest wavelength band, $60-210 \mu \mathrm{m}$, will be covered by the Photodetector Array Camera \& Spectrometer (PACS), which will provide both photometric and spectroscopic observing modes suited to address the key scientific topics of the Herschel mission.

Further author information: (Send correspondence to A.P.)

A.P.: E-mail: alpog@mpe.mpg.de, Telephone: +49 89300003293

Copyright 2008 SPIE. This paper will be published in Proc. SPIE 7010 and is made available as an electronic preprint with permission of the SPIE. One print or electronic copy may be made for personal use only. Systematic or multiple reproduction, distribution to multiple locations via electronic or other means, duplication of any material in this paper for a fee or commercial purposes, or modification of the content of the paper are prohibited. 


\section{INSTRUMENT DESIGN AND SUBUNITS}

The requirements leading to the design of PACS have been discussed previously; ${ }^{1}$ here we briefly summarize the instrument concept. The instrument will offer two basic modes in the wavelength band $60-210 \mu \mathrm{m}$ :

- Imaging dual-band photometry $(60-85 \mu \mathrm{m}$ or $85-125 \mu \mathrm{m}$ and $125-210 \mu \mathrm{m})$ over a field of view of $1.75^{\prime} \times 3.5^{\prime}$, with full sampling of the telescope point spread function (diffraction/wavefront error limited)

- Integral-field line spectroscopy between 57 and $210 \mu \mathrm{m}$ with a resolution of $\sim 175 \mathrm{~km} / \mathrm{s}$ and an instantaneous coverage of $\sim 1500 \mathrm{~km} / \mathrm{s}$, over a field of view of $47^{\prime \prime} \times 47^{\prime \prime}$

Both modes will allow spatially chopped observations by means of an instrument-internal chopper mirror with variable throw; this chopper is also used to alternatively switch two calibration sources into the field of view.

The focal plane sharing of the instrument channels is shown in Fig. 1. The photometric bands, which can be observed simultaneously, cover the same field of view, while the field of view of the spectrometer is offset from the photometer field (Fig. 1). Since photometry and spectroscopy operation are mutually exclusive this has no effect on the observing efficiency. The focal plane unit provides these capabilities through five functional units:

- common input optics with the chopper, calibration sources and a focal plane splitter

- a photometer optical train with a dichroic beam splitter and separate re-imaging optics for the shortwavelength bands $(60-85 \mu \mathrm{m} / 85-125 \mu \mathrm{m})$ and the long-wavelength band $(125-210 \mu \mathrm{m})$, respectively; band-defining filters on a wheel select one of the two short-wavelength bands at a time

- a spectrometer optical train with an image slicer unit for integral field spectroscopy, an anamorphic collimator, a diffraction grating in Littrow mount with associated actuator and position readout, anamorphic re-imaging optics, and a dichroic beam splitter for separation of diffraction orders

- 2 bolometer arrays with cryogenic buffers/multiplexers and a common $0.3 \mathrm{~K}$ sorption cooler

- 2 photoconductor arrays with attached cryogenic readout electronics (CRE).

All subunits have been described in more detail in previous papers; $;^{1,2}$ here we focus on performance tests of subunits, primarily after their integration in the full PACS instrument environment.

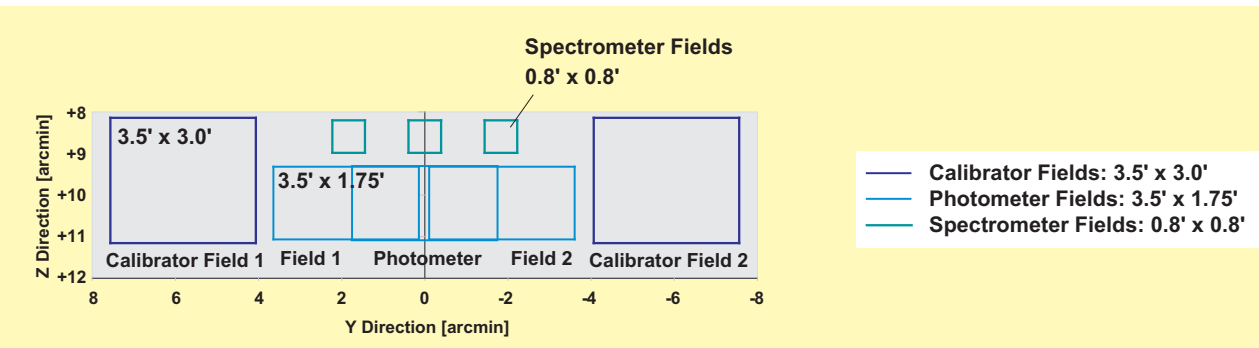

Figure 1. PACS focal plane usage. Long-wavelength and short-wavelength photometry bands cover identical fields of view. The spectrometer field of view is offset in the -z direction. Chopping is done along the $y$ axis (left-right in this view) and also allows observation of the internal calibrators on both sides of the used area in the telescope focal plane. The chopper throw for sky observations is $\pm 1 / 2$ the width of the photometer field such that object and reference fields can be completely separated (photometer field 1 and 2). 

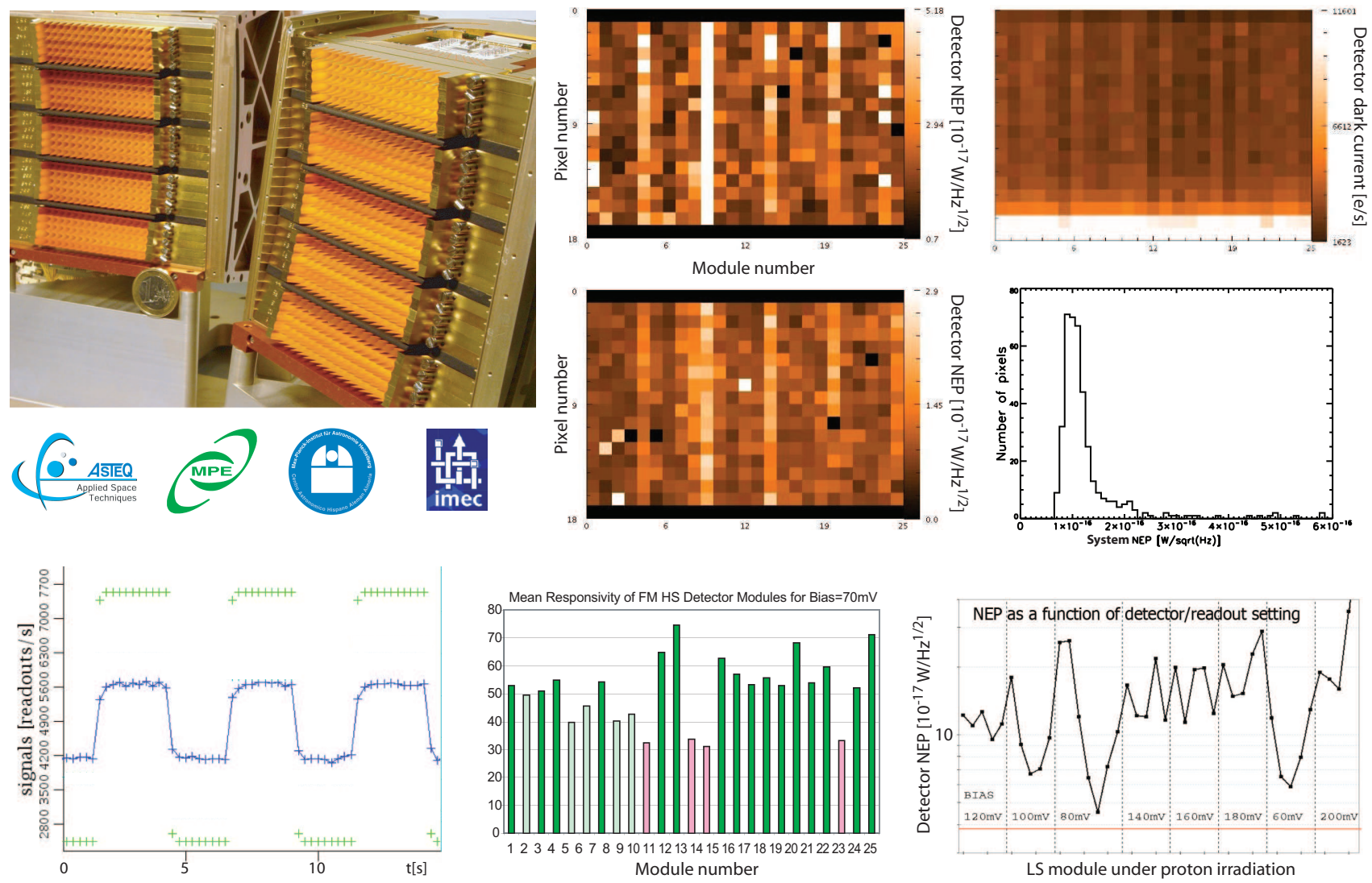

Figure 2. Top left: Fully assembled $25 \times 16$ stressed (back) and unstressed (front) Ge:Ga photoconductor arrays with integrated cryogenic readout electronics. Top center: NEP maps of the unstressed (upper panel) and stressed (lower panel) arrays, referred to the detector entrance plane. Top right: Dark current map (upper panel) and NEP histogram (lower panel) of the unstressed array, referred to the instrument entrance focal plane. Bottom left: Dynamic response of an unstressed detector to a transient signal of $1 / 3$ of the typical background flux (connected symbols) and position readout of the instrument chopper (unconnected symbols), which was used to switch between the two internal calibration sources. Integration time per data point is 1/4 s. Bottom center: Absolute photometric mean response of the 25 modules used for the high-stress flight model array. The modules with the lowest responsivity have been placed in the four corners of the field of view. Bottom right: NEP of an unstressed detector module under high-energy proton irradiation as a function of applied bias and integration reset interval. The horizontal line indicates the performance expected without perturbation.

\subsection{Photoconductor Arrays}

The $25 \times 16$ pixels Ge:Ga photoconductor arrays (Fig. 2) are a completely modular design. 25 linear modules of 16 pixels each are stacked together to form a contiguous, 2-dimensional array. The light cones in front of the actual detector block provide for area-filling light collection in the focal plane. Details of the design of both arrays have been published. ${ }^{3-6}$ Each linear module of 16 detectors is read out by a cryogenic amplifier/multiplexer circuit (CRE) in CMOS technology. ${ }^{7,8}$

Responsivity measurements of both stressed and unstressed modules show sufficiently homogeneous spectral and photometric response within each module and between modules (Fig. 2, bottom center). Absolute responsivities at optimum bias of $11.4 \mathrm{~A} / \mathrm{W}$ for the unstressed detectors and $37.8 \mathrm{~A} / \mathrm{W}$ for the stressed detectors have been determined (median values).

Measurements of the NEP of both arrays after integration into the instrument flight model at characteristic wavelengths and with representative flux levels (Fig. 2, top center) have confirmed the performance measured at module level. The noise distribution (see histogram in Fig. 2) indicates only a small fraction of pixels with excess 

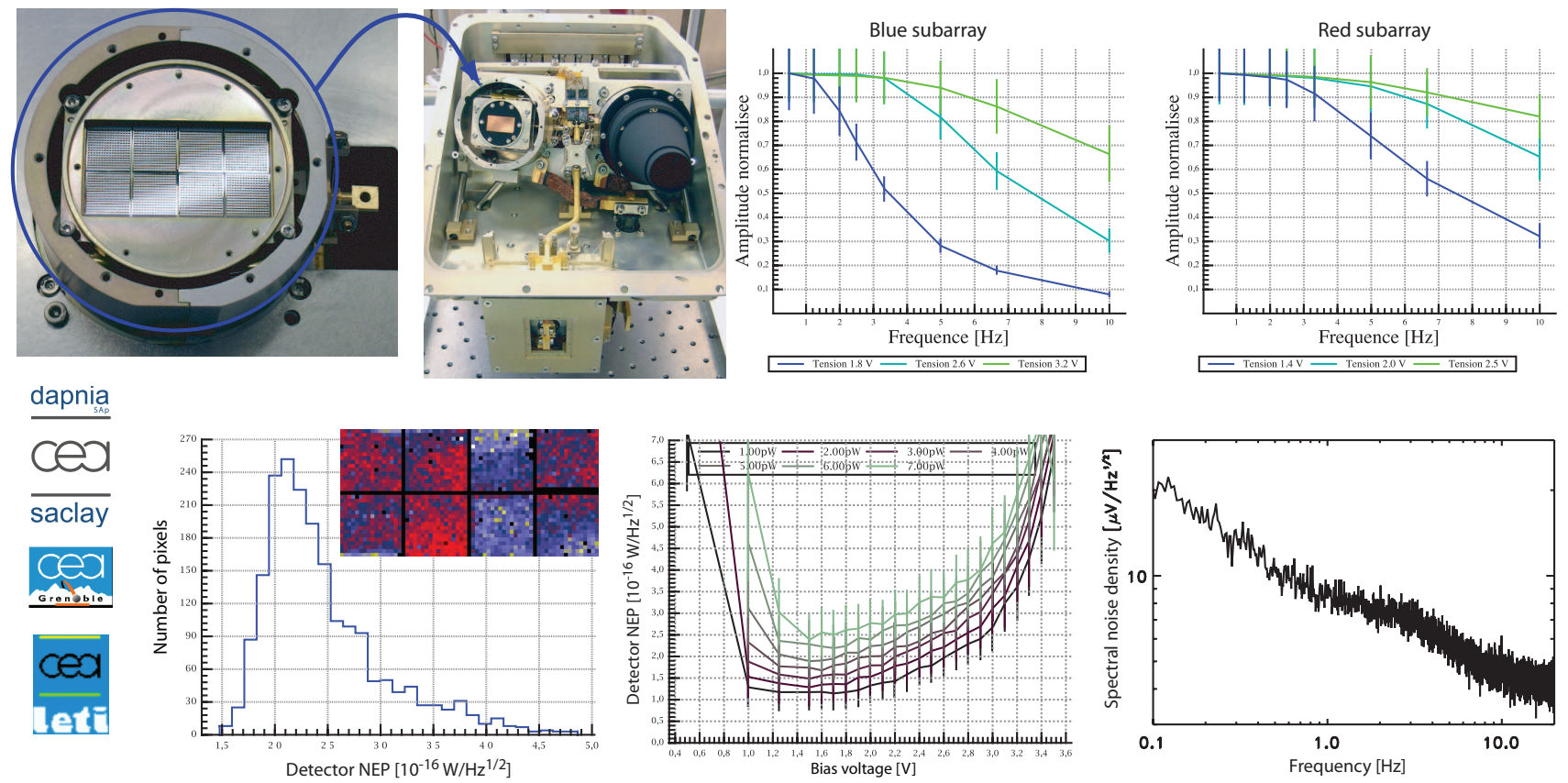

Figure 3. Top left: The PACS photometer subunit. $4 \times 2$ subarrays of $16 \times 16$ pixels, each, form the focal plane of the FM short-wave bolometer assembly (left panel). The $0.3 \mathrm{~K}$ focal plane is suspended from its $2 \mathrm{~K}$ enclosure by Kevlar strings. Close to the right edge of the picture, the thermal interface to the $0.3 \mathrm{~K}$ cooling bar is visible. The complete photometer subunit with the two bolometer assemblies (short-wave, baffle cone removed / long-wave, with baffle cone) and the $0.3 \mathrm{~K}$ sorption cooler is shown in the right panel. Top right: Combined thermal/electrical signal bandwidth of a short-wave (left panel) and a long-wave (right panel) subarray as a function of applied bias voltage. Bottom left: NEP map (inset) and histogram of the short-wave bolometer array under nominal background illumination at nominal bias. Bottom center: NEP as a function of detector bias for a range of background levels. Bottom right: Electrical noise spectrum of the detector/multiplexer combination showing low-frequency excess noise below $1 \mathrm{~Hz}$ and a roll-off frequency which is closely related to the signal bandwidth.

noise. Median NEP values are $8.9 \times 10^{-18} \mathrm{~W} / \mathrm{Hz}^{1 / 2}$ for the stressed and $2.1 \times 10^{-17} \mathrm{~W} / \mathrm{Hz}^{1 / 2}$ for the unstressed detectors, respectively.

The achievable in-orbit performance depends critically on the effects of cosmic rays, in particular, high-energy protons. We have performed proton irradiation tests at the synchrotron source of the Université Catholique de Louvain (Louvain la Neuve, Belgium) on stressed and unstressed detector modules under reasonably realistic conditions in terms of proton flux and energy, FIR background, and metallic shielding by the cryostat. The results indicates that, with optimized detector bias settings and modulation schemes (chopping + spectral scanning), NEPs close to those measured without irradiation can actually be achieved. ${ }^{9}$ Since the dominating effect is a change in responsivity after each hit, fast modulation is essential. We have tested the transient response of the photoconductors by chopping between the two internal PACS calibration sources (Fig. 2, bottom left). Between two consecutive integration ramps of the detector readout with a $1 / 4 \mathrm{~s}$ duration, more than $80 \%$ of the stationary response is achieved.

\subsection{Bolometer Arrays}

The PACS bolometers are filled arrays of square pixels which allow instantaneous beam sampling. $4 \times 2$ monolithic sub-arrays of $16 \times 16$ pixels are tiled together to form the short-wave focal plane array (Fig. 3, top center). In a similar way, 2 sub-arrays of $16 \times 16$ pixels are tiled together to form the long-wave focal plane array. The subarrays are mounted on a $0.3 \mathrm{~K}$ carrier which is thermally isolated from the surrounding $2 \mathrm{~K}$ structure. The buffer/multiplexer electronics is split in two levels; a first stage is part of the indium-bump bonded back plane of the focal plane arrays, operating at $0.3 \mathrm{~K}$, and a buffer stage running at $2 \mathrm{~K}$. The multiplexing readout samples 

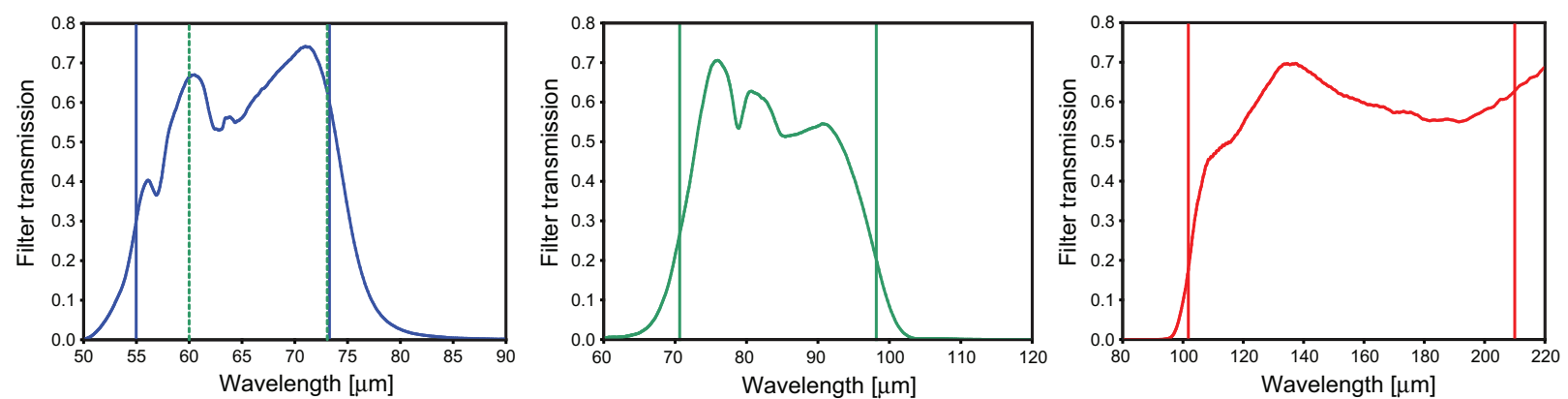

Figure 4. Filter transmission of the spectrometer filters. The graphs represent the overall transmission of the combined filters in each branch of the instrument. The nominal band limits are indicated by the vertical lines. Left: $55-73 \mu m$ spectrometer band. This filter band was desigend for use in the third grating order, but can be used in the "extended second order" mode down to $60 \mu \mathrm{m}$, as indicated by the dashed line. Centre: $71-98 \mu \mathrm{m}$ spectrometer band (second order). Right: $102-210 \mu \mathrm{m}$ spectrometer band (first order). Note: Tolerances intrinsic to the filter manufacturing process have led to an effective gap in wavelength coverage between 98 and $102 \mu \mathrm{m}$.

each pixel at a rate of $40 \mathrm{~Hz}$ or $20 \mathrm{~Hz}$. Details on the bolometer design have been published. ${ }^{10-13}$ Both array assemblies are mounted in a subunit of the FPU (Fig. 3, top left) together with the $0.3 \mathrm{~K}$ cooler ${ }^{14}$ which provides uninterrupted operation for two days. The post-detection bandwidth (thermal/electrical) of the bolometers is $\sim 5 \mathrm{~Hz}$ at nominal bias and can be traded off against NEP; the noise of the bolometer/readout system has a strong $1 / \mathrm{f}$ component such that a clear $1 / \mathrm{f}$ "knee" frequency cannot be defined (Fig. 3, bottom right). To achieve optimum sensitivity, observations have to be executed such that the signal modulation due to chopping or scanning falls primarily in the frequency band from $1 \mathrm{~Hz}$ to $5 \mathrm{~Hz}$. Measurements of the bolometer NEP as a function of electrical bias for a range of background loads (Fig. 3, bottom center) show that there is a potential for improvement in NEP should the Herschel telescope background be lower than specified in the requirements.

\subsection{Filter Chains}

The filters used in PACS in transmission or reflection have been measured individually in our FTS before integration. The optical efficiencies of the filter chains have then been calculated by multiplying the individual filter efficiencies. The spectrometer filter chain efficiencies are shown in Fig. 4, the photometer band efficiencies, obtained by multiplying all filter efficiencies and the bolometer efficiency in each band, are shown in Fig. 8

\section{INSTRUMENT LEVEL TEST RESULTS}

A wide range of tests has been performed on components, subunits, and the full instrument in order to characterise and calibrate the instrument under flight-representative conditions, as much as possible, on ground. We present results with the most direct impact on the performance of the instrument.

\subsection{Instrument Point Spread Function}

Measurements of the instrument PSF were performed in all three photometric bands as well as in both branches of the spectrometer. The telescope simulator used for the instrument level tests has a significantly smaller wavefront error than the Herschel telescope specification. We expect a very similar PSF in orbit; the wavefront error of the telescope is expected to be small enough that it will mostly lead to some loss of power in the central peak of the PSF but not significantly increase its width.

\subsubsection{Photometer Point Spread Function}

Measurements of the photometer PSF in the $70 \mu \mathrm{m}$ band, done with a pinhole source at the entrance to our telescope simulator optics, are shown in Figure 5. This is the most critical band since it should deliver the narrowest PSF.

The measurements in all three bands agree well with the predicted profiles based on a convolution of the pinhole source with the diffraction by the optics and the pixel size. 

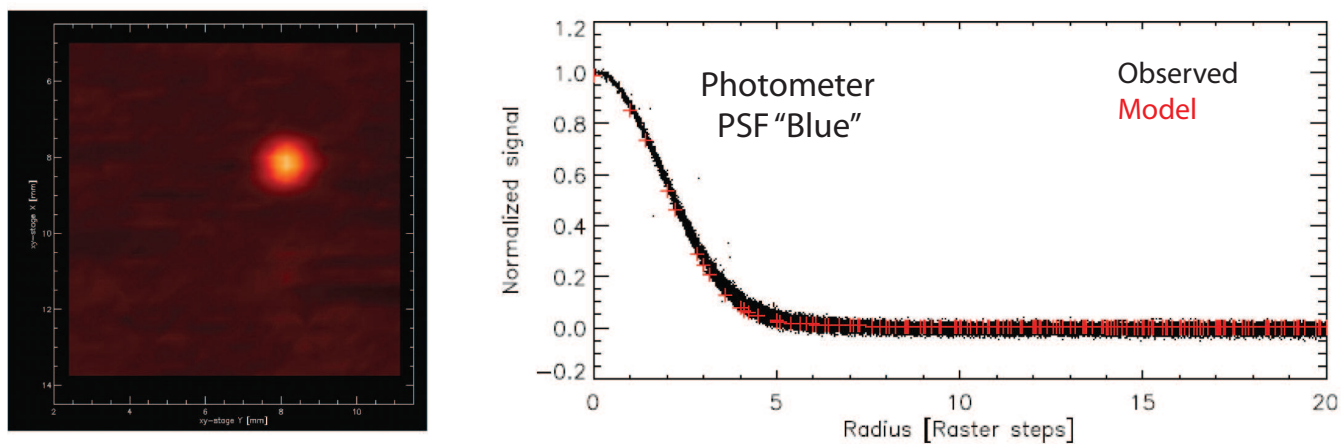

Figure 5. Measurement of the photometer PSF in the $70 \mu \mathrm{m}$ band. The source was a hole mask, which was scanned across the field of view in 1/3 pixel steps. The left panel shows the image of the scanned source as seen by one pixel. The right panel shows a radial cut of the PSF obtained from all pixels. The central peak appears slightly wider than the model (red crosses), but there is no indication of unexpected large scale wings.
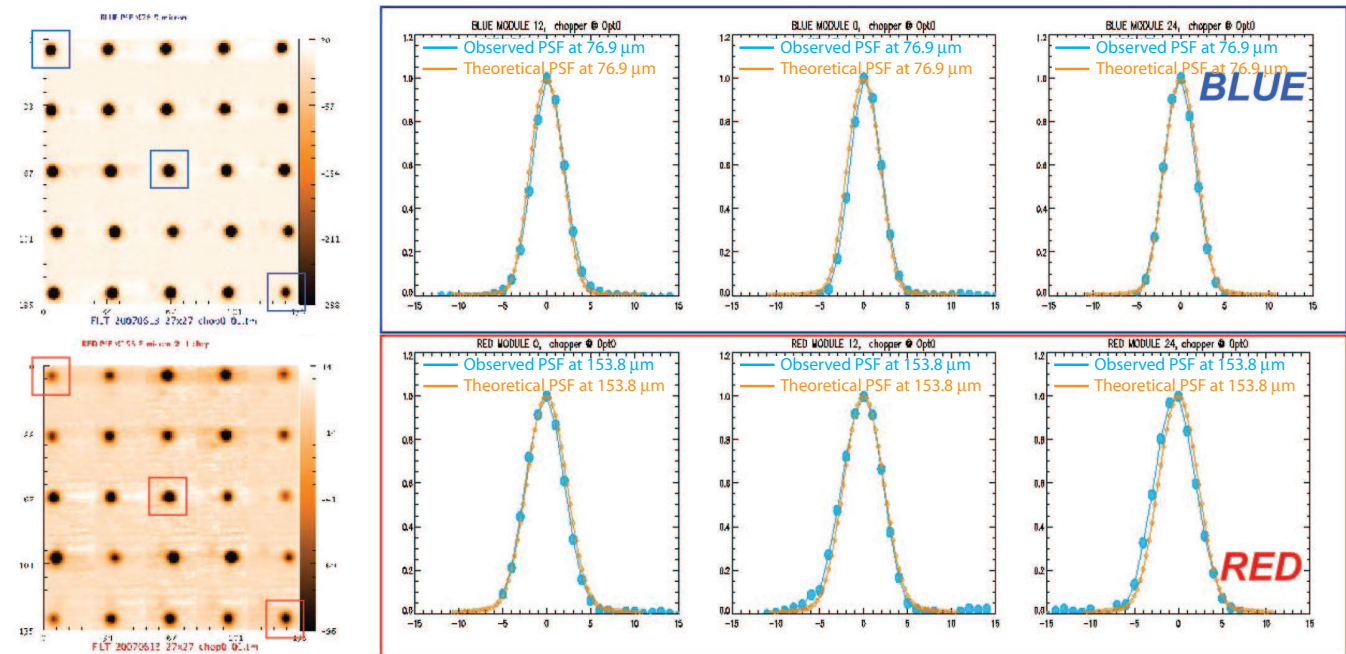

Figure 6. Measurement of the spectrometer PSF in the "blue" and "red" branches. The source was a hole mask, which was scanned across the field of view in 1/3 pixel steps. The left panels show the (spectrally collapsed) images of the scanned source as seen by each spatial pixel. The right panels show cuts through three selected pixels, as indicated by the boxes in the left panels, in both spectrometer branches.

\subsubsection{Spectrometer Point Spread Function}

Measurements of the spectrometer PSF in the "blue" and "red" branches, done with a pinhole source at the entrance to our telescope simulator optics, are shown in Figure 6. Since the source had no spectral features, each of the $5 \times 5$ spatial elements in our field of view has been spectrally collapsed, to increase the signal-to-noise ratio.

The measurements in all three bands agree well with the predicted profiles based on a convolution of the pinhole source with the diffraction by the optics and the pixel size.

\subsubsection{Spectrometer Spectral Calibration and Line Profile}

For spectral calibration of the instrument a water vapour absorption cell was used. With the large number of available lines, an absolute wavelength calibration accuracy of $10 \%$ to $20 \%$ of a spectral resolution element has been achieved over the entire PACS wavelength range. 

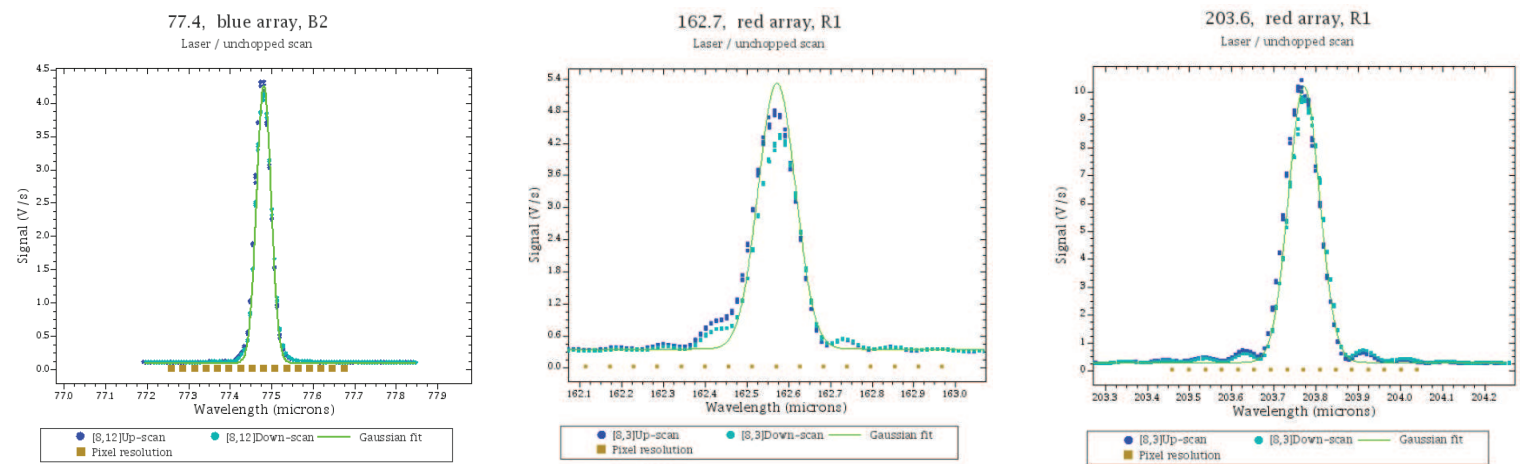

Figure 7. Measurements of the spectrometer instrument profile at one wavelength in the "blue" and two wavelengths in the "red" branch. Gaussian fits to each profile have been used to derive the spectral resolution (FWHM) for comparison with the calculated spectral resolution of the instrument (see Fig. 9).

The spectral profile of the instrument has been checked at a few wavelengths with an optically pumped FIR gas laser ${ }^{15}$ as a source of monochromatic light. The measured line profiles (Fig. 7) agree well with the calculated line widths (Fig. 9).

\section{SYSTEM PERFORMANCE PREDICTION}

Based on the results from the Instrument Level Tests and our tests of FM components/subunits and our present knowledge of the Herschel satellite, the performance of the entire system can be predicted through a detailed instrument model.

The system sensitivity of the instrument at the telescope depends mainly on the optical efficiency, i.e. the fraction of light from an astronomical source arriving at the telescope that actually reaches the detector, on the photon noise of the thermal background radiation from the telescope or from within the instrument, and on detector/electronics noise.

\subsection{Optical Efficiency}

The system optical efficiency has been modeled to the following level of detail:

- Telescope efficiency: The fraction of the power of a point source in the central peak of the point spread function is modeled in terms of absorption/obstruction, diffraction, and geometrical wave front errors $(6 \mu \mathrm{m}$ r.m.s.), which have been assumed to occur as spherical aberration.

- Chopper: Errors/jitter in the chopper throw and the duty cycle (> 80\%) are considered.

- Mirrors and filters: scatter/absorption losses - excluding diffraction - on each reflection by a mirror (1\%) and efficiencies of filters/dichroics (as measured for the FM filters individually) are taken into account.

- Diffraction: An end-to-end diffraction analysis with the physical optics package GLAD 4.5 has been carried out for the spectrometer, where the image slicer is the most critical element of the PACS optics, ${ }^{16,17}$ and a simplified analysis for the less critical photometer as well as the effect of diffraction/vignetting by the entrance field stop and Lyot stop have been included.

- Grating efficiency: The grating has been analysed and optimised with a full electromagnetic code; ${ }^{16}$ the validity of this code has been confirmed by FTS measurements on a grating sample made by the manufacturer of the FM grating. 


\subsection{Detectors}

The NEP of the Ge:Ga photoconductor system is calculated over the full wavelenegth range of PACS based on the CRE noise and peak quantum efficiency determination at detector module level for the high-stress detectors. For the low-stress detectors we assume the same peak quantum efficiency. The quantum efficiency as a function of wavelength for each detector can be derived from the measured relative spectral response function. To account for cosmic ray effects we assume an effective loss of observing time of $20 \%$ from rejected integration ramps.

For the bolometers, we use the "end-to-end" system NEPs gained in all three photometer bands during the instrument level tests for both, the "blue" and "red" focal planes under realistic photon background.

\subsection{System Sensitivity}

For the calculation of the system sensitivity we have included our present best knowledge of all components in the detection path as described above. The results for photometry and spectroscopy are shown in Figs. 8 and 9.
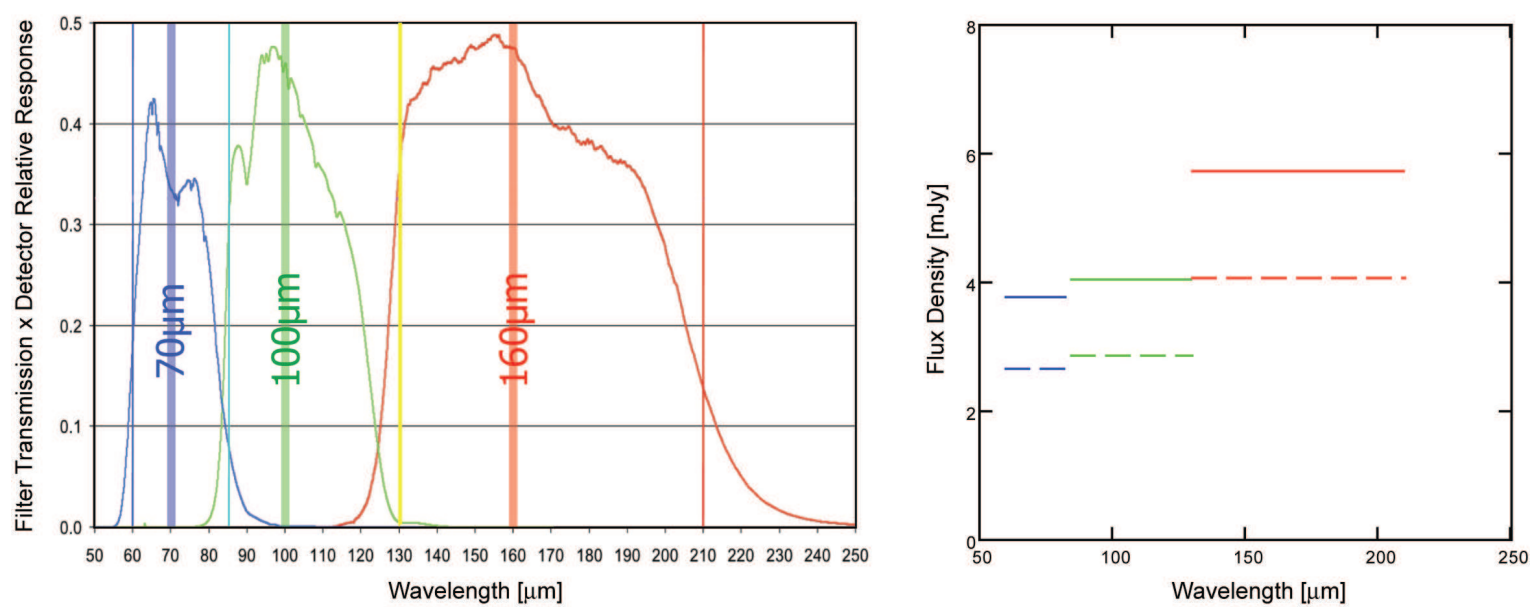

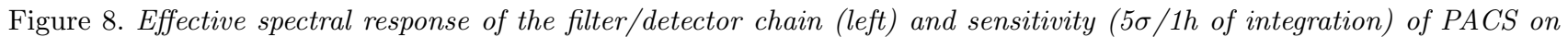
Herschel for point source detection in photometry mode (right. The solid lines represent chopped observations where only half of the integration time is spent on the source. The dashed lines represent modulation techniques like on-array chopping or line scans where the source is on the array all the time.

It needs to be stressed that these figures must be considered somewhat preliminary, since the instrument level tests of the Flight Model could not fully mimic the satellite environment. In some areas (e.g. "red" bolometer detector) we may foresee some improvement compared to the achieved performance used in the system model, in other areas, in particular, the behaviour of the photoconductors under cosmic ray irradiation in the Herschel cryostat, predictions seem less reliable, and more severe adjustments of the sensitivity may occur after the satellite has reached its orbit. The numbers presented here do not contain satellite/AOT overheads, e.g., for slewing, calibration times, or additional "penalties". Exact numbers, therefore, can only be obtained through the HSPOT observing time calculator, which can be downloaded from the Herschel Science Centre site (http://herschel.esac.esa.int).

\section{OBSERVING WITH PACS}

\subsection{AOT concept}

Either the photometer or the spectrometer will be used during dedicated Observation Days (OD) of 21 hours. The reason for this is to allow uninterrupted observations with the photometer to optimize the time spent on recycling the photometer cooler, which takes about 2 hours, during a daily telecommunication period of 3 hours per day. As the hold time of the cooler will probably be approximately 48 hours, the photometer might even be used for two consecutive ODs. 

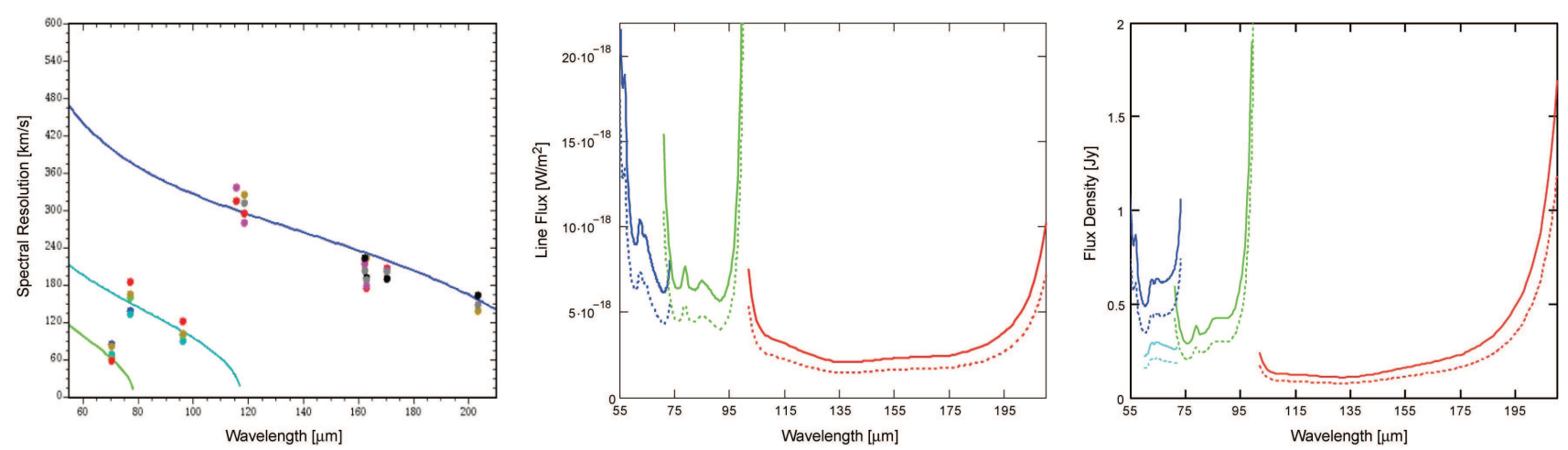

Figure 9. Spectral resolution as predicted and verified at several, selected wavelengths (left) and sensitivity (5 $/ 1 \mathrm{~h}$ of integration) of the PACS instrument on Herschel for point source detection in spectroscopy mode (right). For spectroscopy we distinguish between line detection, where we assume that the astronomical line is unresolved at the respective resolving power of the instrument (center), and continuum detection where the quoted sensitivity is reached for each spectral resolution element over the instantaneous spectral coverage of the instrument. The solid lines represent chopped observations where only half of the integration time is spent on the source. The dashed lines represent modulation techniques like on-array chopping or line scans where the source is on the array all the time.

The Herschel observations are organized around standardized observing procedures, called AOTs (for Astronomical Observation Template). Four different AOTs have been defined and implemented to perform astronomical observations with PACS: two for the spectrometer, one generic for photometry/mapping with the photometer, and one having both SPIRE and PACS observing in parallel with their respective photometers.

\subsection{Spectrometer AOTs}

Two different observation schemes are offered with the PACS spectrometer: line and range spectroscopy.

- Line spectroscopy mode: a limited number of relatively narrow emission or absorption lines can be observed for either a single spectroscopic FOV $\left(0.78^{\prime} \mathrm{x} 0.78^{\prime}\right)$ or for a larger map. Background subtraction is achieved either through standard chopping/nodding, for faint/compact sources, or through 'wavelength-switching' techniques for line measurement of the grating mechanism of bright extended sources.

- Range spectroscopy mode: this is a more flexible and extended version of the line spectroscopy mode, where a freely defined wavelength range is scanned by stepping through the relevant angles of the grating, synchronized with the chopper. Both arrays are used at a time.

\subsubsection{Line spectroscopy}

This AOT is designed to observe one or several unresolved or narrow spectral line features, within a fixed wavelength range, varying from 0.35 to $1.8 \mu \mathrm{m}$ depending on the wavelength and the grating order.

Only lines in the first $(102-210 \mu \mathrm{m})$ and second order $(73-98 \mu \mathrm{m})$, or first and third order $(55-73 \mu \mathrm{m})$ can be observed within a single AOR, to avoid filter wheel movements. If lines of second and third grating order are to be observed on the same target at the same time, two AORs shall be concatenated. Depending on the requested wavelength/grating order, only the data of one of the two detector arrays is normally of interest to the observer.

The specified wavelength and its immediate neighborhood are observed for each chopper and grating position. For improved flat-fielding, especially for long integrations, the grating is scanned by a number of discrete steps around a specified centre position such that drifts in the detector responsivity between individual pixels are eliminated. The principle of line spectroscopy is illustrated in Figure 10.

These grating scans provide, for each line and for each of the 5 by 5 spatial pixels, a short spectrum with a resolving power of $\sim 1700$ in its highest resolution covering $\sim 1500 \mathrm{~km} / \mathrm{s}$, but dependent on the wavelength and order. 


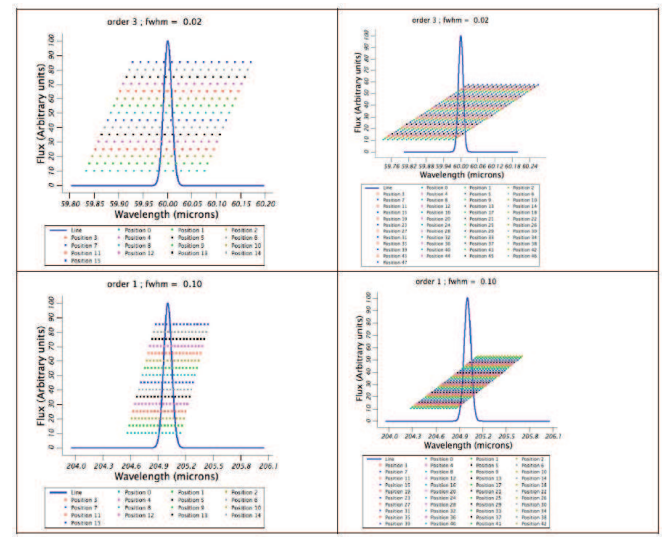

\section{AOTs in photometry channel}

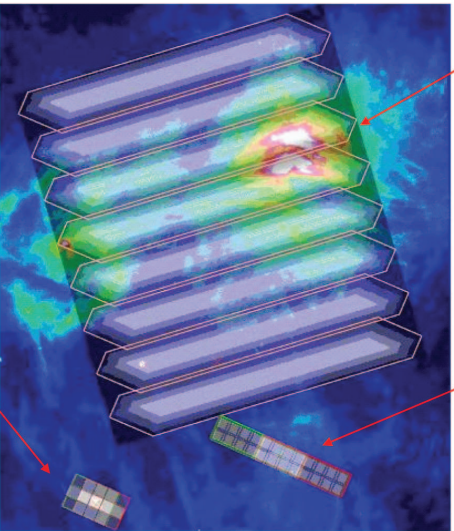

Extended source

Options are Scan (shown) or chopped Raster

Maximum size 4-deg

3 scan speeds or fixed chopping

photometry:
4-positions
2 chop/nod cycles
Repeat basic cycle
to gain more
sensitivity

\section{Small source} photometry:

Small $2 \times 2$ raster 200"x100" FOV Dither to cover inter-matrix gaps

Figure 10. Left Panel: Visualization of the line scan AOT on an unresolved PACS line (here given by a Gaussian). The grating step used is the nominal one currently coded in AOT design. The left-hand side shows results for 16 grating steps (bright lines chopping-nodding mode case) the right-hand side shows the same for nominal grating positions (standard "faint lines" chopping-nodding) with 48 grating steps. Top row is for a blue line at $60 \mu \mathrm{m}$; bottom row is for a red line at $205 \mu \mathrm{m}$. Right Panel: Visualization of the 4 photometer AOTs (courtesy NHSC).

Up to 10 lines can be studied within one observation. The relative sensitivity between the lines is controlled by using the line repetition factor, in the line editor of the "wavelength settings" in HSpot, that allows one to repeat a line scan several times.

Background subtraction is achieved either through standard chopping/nodding (for faint/compact sources) with a selected chopper throw of 1,3 or 6 arcmin, or through "frequency-switching" techniques (for line measurements of bright extended sources) of the grating mechanism in combination with the mapping mode. The mapping mode can also be used in chopping/nodding but the map size is then limited to 6 arcmin (the larger chop throw) to avoid self-chopping. Two flavors of the chopping/nodding are available: a default one with 48 grating steps up and down and a faster version for bright lines only with 16 steps.

\subsubsection{Range Spectroscopy}

Similarly to the line scan spectroscopy mode, this AOT allows one to observe one or several spectral line features (up to ten), but the user can freely specify the covered wavelength range.

This AOT is mainly intended for observations of cover rather limited wavelength ranges up to a few microns in high sampling mode (see below) to study broad lines (larger than a few hundreds $\mathrm{km} / \mathrm{s}$ ), whose wings would not be covered sufficiently in Line Spectroscopy AOT, or a set of closely spaced lines.

The Range Spectroscopy AOT is also suited for covering larger wavelength ranges up to the entire bandwidth of PACS (in SED mode) with lower sampling density, as otherwise integration times would get quickly prohibitive. In this mode a given wavelength is seen only by two spectral pixels.

The use of the chopping/nodding is imposed by the design of the AOT, except in mapping mode where, instead, an off position can be defined if chopping/nodding is de-selected. In this case only chopping is performed on one of the calibration source and the background subtraction shall be done with OFF position. The chopping/nodding uses the same pattern as in line spectroscopy, with a 3-positions chopping/nodding and same chopper throws to eliminate inhomogeneities in the telescope and sky background. As in line spectroscopy, only ranges in first $(102-210 \mu \mathrm{m})$ and second order $(71-98 \mu \mathrm{m})$, or first and third order $(55-73 \mu \mathrm{m})$ are allowed within a single AOR.

\subsection{Photometer AOT}

Four generic observing modes are offered to observe with the photometer (Figure 10). The first three pointed and raster modes make use of chopping at a frequency of $1.25 \mathrm{~Hz}$ (i.e. 4 averaged frames per chopper plateau), to remove the high telescope background and mitigate the effects of $1 / \mathrm{f}$ noise and detector gain drifts. In scan mapping mode the modulation is provided by the spacecraft motion, hence no chopping is applied. 


\subsubsection{Point-source mode}

Point-source photometry: This mode is targeted at observations of isolated, "point-like" sources (smaller than one blue matrix). A typical use of this mode is for point-source photometry.

It makes use of a classical 4-positions on-array chopping, with dithering option, along the Y-axis combined with nodding along the Z-axis to compensate for the different optical paths. The chopper is used to alternate the source between the left and right part of the array, and the satellite nodding is used to alternate it between the top and bottom part of the array, so that the target is always on the array.

To achieve photometry of fainter sources, the number of nod cycles is increased with the 'repetition factor' in the 'observing mode settings' to improve the sensitivity and reach fainter flux levels. The sensitivity scales with the inverse of the square root of integration time and repetition factor.

The minimal observing time is about $5.5 \mathrm{~min}$ with a predicted point-source sensitivity of $15-20 \mathrm{mJy}(5 \sigma)$

\subsubsection{Small-source mode}

Small source photometry: This mode is tailored to observe sources that are smaller than the array size, yet larger than a single matrix. To be orientation independent, this means sources that fit in circle of 1.5 arcmin diameter. This mode uses also chopping and nodding, but this time the source cannot be kept on the array at all times.

In this mode, a small $2 \mathrm{x} 2$ raster with small step size $(17 \mathrm{arcsec})$ is performed to observe the target, with a classical 3-positions chopping/nodding for each raster position, spending 1 minute on each raster position (both on the nod-on and nod-off). Therefore only half of the science time is actually used for on-source integration, in contrast to the point-source photometry observing mode. With the pattern of gaps between matrices, the $2 \mathrm{x} 2$ raster map allows one to recover the signal lost between pixels. This scheme also adds the advantage of a larger fully-covered area. The parameters of this raster (i.e. the displacement in both directions, nod and chop throws) are fixed and not left to the observer's choice.

The minimal observing time is about $15 \mathrm{~min}$ with a predicted point-source sensitivity of $10-15 \mathrm{mJy}(5 \sigma)$

\subsubsection{Raster mode}

In raster mapping the spacecraft goes through a rectangular grid of pointings in instrument reference frame, spending 1 minute per raster position, and chopping by one full array ( 3.5 arcmin) along the long axis of the detector.

It is best suited for covering a limited area in the sky of up to 15'x15'. The sensitivity of the map is adjusted by the number of map repetitions.

\subsubsection{Scan mode}

The scan mapping is the default mode to cover large areas in the sky for galactic as well as extragalactic surveys. Scan maps are performed by slewing the spacecraft at a constant speed (10, 20 or 60 arcsec/s) along parallel lines, without chopping, the signal modulation being provided by the spacecraft motion.

The highest speed (default value) is envisaged for galactic surveys only, with a significant degradation of the PSF due to the limited signal bandwidth of the detection chain.

The slow scan speed shall be used for extragalactic surveys. It allows one to cover an area of 1 square degree in about three hours. The PSF degradation and smearing due to the scanning should be almost negligible with the two lowest scan speeds, according to simulations.

Two scan maps of the same area with orthogonal coverage shall be performed to mitigate striping effects due to $1 / \mathrm{f}$ noise or drifts, in order to recover extended emission

PACS scan maps can be performed either in the instrument reference frame or in sky coordinates.

In all photometer observing modes, dual-band imaging observations are performed, either in the blue $(70 \mu \mathrm{m})$ and red $(160 \mu \mathrm{m})$ bands or in the green $(100 \mu \mathrm{m})$ and red $(160 \mu \mathrm{m})$ bands. Further details are given in ${ }^{18}$ and can also be found on the Herschel Science Centre site (http://herschel.esac.esa.int). 


\section{ACKNOWLEDGMENTS}

This work is supported by the following funding agencies: ASI (Italy), BMVIT (Austria), CEA/CNES (France), DLR (Germany), ESA-PRODEX (Belgium), and CDT (Spain).

\section{REFERENCES}

[1] Poglitsch, A., Waelkens, C., and Geis, N. in [IR Space Telescopes and Instruments], Mather, J., ed., Proc. SPIE 4850, 662-673 (2003).

[2] Poglitsch, A., Waelkens, C., Bauer, O. H., Cepa, J., Henning, T. F., van Hoof, C., Katterloher, R., Kerschbaum, F., Lemke, D., Renotte, E., Rodriguez, L., Royer, P., and Saraceno, P. in [Optical, Infrared, and Millimeter Space Telescopes], Mather, J. C., ed., Proc. SPIE 5487, 425-436 (2004).

[3] Kraft, S., Frenzl, O., Charlier, O., Cronje, T., Katterloher, R. O., Rosenthal, D., Groezinger, U., and Beeman, J. W. in [UV, Optical, and IR Space Telescopes and Instruments], Breckinridge, J. B. and Jakobsen, P., eds., Proc. SPIE 4013, 233-243 (2000).

[4] Kraft, S., Merken, P., Creten, Y., Putzeys, J., van Hoof, C., Katterloher, R. O., Rosenthal, D., Rumitz, M., Groezinger, U., Hofferbert, R., and Beeman, J. W. in [Sensors, Systems, and Next-Generation Satellites V], Fujisada, H., Lurie, J. B., and Weber, K., eds., Proc. SPIE 4540, 374-385 (2001).

[5] Rosenthal, D., Beeman, J. W., Geis, N., Groezinger, U., Hoenle, R., Katterloher, R. O., Kraft, S., Looney, L. W., Poglitsch, A., Raab, W., and Richter, H. in [Proceedings FIR, Submm \& $\mathrm{mm}$ Detector Technology Workshop], Wolf, J., Farhoomand, J., and McCreight, C., eds., NASA/CP- 211408 (2002).

[6] Poglitsch, A., Katterloher, R., Hoenle, R., Beeman, J., Haller, E., Richter, H., Groezinger, U., Haegel, N., and Krabbe, A. in [Millimeter and Submillimeter Detectors for Astronomy], Phillips, T. and Zmuidzinas, J., eds., Proc. SPIE 4855, 115-128 (2003).

[7] Charlier, O. in [UV, Optical, and IR Space Telescopes and Instruments], Breckinridge, J. B. and Jakobsen, P., eds., Proc. SPIE 4013, 325-332 (2000).

[8] Creten, Y., Merken, P., Putzeys, J., and van Hoof, C. in [Proceedings FIR, Submm \&3 mm Detector Technology Workshop], Wolf, J., Farhoomand, J., and McCreight, C. R., eds., NASA/CP- 211408 (2002).

[9] Katterloher, R., Barl, L., Poglitsch, A., Royer, P., and Stegmaier, J. in [Millimeter and Submillimeter Detectors and Instrumentation for Astronomy III], Zmuidzinas, J., Holland, W. S., Withington, S., and Duncan, W. D., eds., Proc. SPIE 6275, 627515 (2006).

[10] Agnese, P., Buzzi, C., Rey, P., Rodriguez, L., and Tissot, J.-L. in [Infrared Technology and Applications XXV], Andresen, B. F. and Scholl, M. S., eds., Proc. SPIE 3698, 284-290 (1999).

[11] Agnese, P., Cigna, C., Pornin, J.-L., Accomo, R., Bonnin, C., Colombel, N., Delcourt, M., Doumayrou, E., Lepennec, J., Martignac, J., Reveret, V., Rodriguez, L., and Vigroux, L. in [Millimeter and Submillimeter Detectors for Astronomy], Phillips, T. and Zmuidzinas, J., eds., Proc. SPIE 4855, 108-114 (2003).

[12] Simoens, F., Agnese, P., Béguin, A., Carcey, J., Cigna, J.-C., Pornin, J.-L., Rey, P., Vandeneynde, A., Rodriguez, L., Boulade, O., Lepennec, J., Martignac, J., Doumayrou, E., Reveret, V., and Vigroux, L. in [Millimeter and Submillimeter Detectors for Astronomy II], Zmuidzinas, J., Holland, W. S., and Withington, S., eds., Proc. SPIE 5498, 177-186 (2004).

[13] Billot, N., Agnèsel, P., Auguères, J.-L., Béguin, A., Boulade, O., Cara, C., Cloué, C., Doumayrou, E., Duband, L., Horeau, B., le Mer, I., Lepennec, J., Martignac, J., Okumura, K., Revéret, V., Sauvage, M., Simoens, F., and Vigroux, L. in [Space Telescopes and Instrumentation I: Optical, Infrared, and Millimeter], Mather, J. C., ed., Proc. SPIE 6265, 9B (2006).

[14] Duband, L. and Collaudin, B. Cryogenics 39, 659-663 (1999).

[15] Inguscio, M., Moruzzi, G., Evenson, K., and Jennings, D. J.Appl.Phys. 60, 161-191 (1986).

[16] Poglitsch, A., Waelkens, C., and Geis, N. in [Infrared Spaceborne Remote Sensing VII], Scholl, M. S. and Andresen, B. F., eds., Proc. SPIE 3759, 221-233 (1999).

[17] Looney, L., Raab, W., Poglitsch, A., and Geis, N. ApJ 597, 628-643 (2003).

[18] Poglitsch, A. and Altieri, B. in [Astronomy in the submillimeter and far infrared domains with the Herschel Space Observatory], Pagani, L., ed., EAS Publication Series 34, (in press) (2008). 